\title{
Necessary and Sufficient Conditions for Input-Output Finite-Time Stability of Linear Time-Varying Systems
}

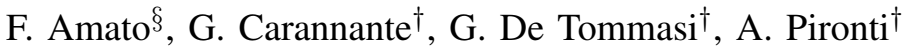

\begin{abstract}
In the recent paper [3] a sufficient condition for Input-Output Finite-Time stability (IO-FTS) has been provided by the authors. By using an approach based on Reachability Gramian theory, in this paper we show that such condition, which requires the solution of a feasibility problem involving differential linear matrix inequalities (DLMIs), is also necessary for IO-FTS when the class of system inputs belong to $\mathcal{L}_{2}$. A numerical example illustrates the proposed technique.
\end{abstract}

Keywords: Linear systems; time-varying systems; Reachability Gramian; IO-FTS; DLMIs.

\section{INTRODUCTION}

The concept of input-output finite time stability (IO-FTS) has been recently introduced in [3]; roughly speaking, a system is said to be IO-FTS if, given a class of norm bounded input signals defined over a specified time interval $T$, the outputs of the system do not exceed an assigned threshold during $T$.

In order to correctly frame the definition of IO-FTS in the current literature, we recall that a system is said to be IO $\mathcal{L}_{p}$-stable [11, Ch. 5] if for any input of class $\mathcal{L}_{p}$, the system exhibits a corresponding output which belongs to the same class. The main differences between classic IO stability and IO-FTS are that the latter involves signals defined over a finite time interval, does not necessarily require the inputs and outputs to belong to the same class, and that quantitative bounds on both inputs and outputs must be specified. Therefore, IO stability and IO-FTS are independent concepts. Furthermore, while IO stability deals with the behavior of a system within a sufficiently long (in principle infinite) time interval, IO-FTS is a more practical concept, useful to study the behavior of the system within a finite (possibly short) interval, and therefore it finds application whenever it is desired that the output variables do not exceed a given threshold during the transients, given a certain class of input signals.

It is important to remark that the definition of IO-FTS given in [3] is fully consistent with the definition of (state) FTS given in [8], where the state of a zero-input system, rather than the intput and the output, are involved. Sufficient conditions for (state) FTS and finite time stabilization (the corresponding design problem) have been provided in the control literature in the last fifteen years, see for example [5],

\footnotetext{
$\S$ F. Amato is with the School of Computer Science and Biomedical Engineering, Università degli Studi Magna Græcia di Catanzaro, Via Tommaso Campanella 115, 88100 Catanzaro, Italy.

$\dagger$ G. Carannante, G. De Tommasi and A. Pironti are with the Dipartimento di Informatica e Sistemistica, Università degli Studi di Napoli Federico II, Via Claudio 21, 80125 Napoli, Italy.
}

[4], [10], [12] in the context of linear systems, [6], [15] in the context of impulsive and hybrid systems.

In [3] two sufficient conditions for IO-FTS were provided, for the class of $\mathcal{L}_{2}$ inputs and the class of $\mathcal{L}_{\infty}$ inputs respectively. Both conditions required the solution of a feasibility problem involving differential linear matrix inequalities (DLMIs).

The main contribution of this paper shows that, in the important case of $\mathcal{L}_{2}$ inputs, the condition given in [3] is also necessary. To prove this result, a machinery involving the Reachability Gramian is used. More precisely, we shall prove that, if a given system is IO-FTS, then a generalized Lyapunov differential equation involving the Reachability Gramian admits a positive definite solution, which satisfies over the time interval of interest a certain condition. This in turn is proven to imply the condition for IO-FTS given in [3]; then we can conclude that the two conditions are equivalent each other and both are equivalent to IO-FTS.

The paper is organized as follows: in Section II the problem we deal with is precisely stated and some preliminary results are given. In Section III the main result, namely a theorem containing two necessary and sufficient conditions for IO-FTS, is provided. In Section IV a numerical example is provided to illustrate the proposed approach. Eventually, in Section V, some concluding remarks are given.

Notation. Given a vector $v \in \mathbb{R}^{n}$ and a matrix $A \in \mathbb{R}^{n \times n}$, we will denote with $|v|$ the euclidian norm of $v$, and with $|A|$ the induced matrix norm

$$
|A|=\sup _{v \neq 0} \frac{|A v|}{|v|} .
$$

Given the set $\Omega=\left[t_{0}, t_{0}+T\right]$, with $t_{0} \in \mathbb{R}$ and $T>0$, the symbol $\mathcal{L}_{p}(\Omega)$ denotes the space of vector-valued signals for which ${ }^{1}$

$$
s(\cdot) \in \mathcal{L}_{p}(\Omega) \Longleftrightarrow\left(\int_{\Omega}|s(\tau)|^{p} d \tau\right)^{\frac{1}{p}}<+\infty .
$$

Given a symmetric positive definite matrix valued function $R(\cdot)$, bounded on $\Omega$, and a vector-valued signal $s(\cdot) \in$ $\mathcal{L}_{p}(\Omega)$, the weighted signal norm

$$
\left(\int_{\Omega}\left[s^{T}(\tau) R(\tau) s(\tau)\right]^{\frac{p}{2}} d \tau\right)^{\frac{1}{p}},
$$

will be denoted by $\|s(\cdot)\|_{p, R}$. If $p=\infty$

$$
\|s(\cdot)\|_{\infty, R}=\operatorname{ess} \sup _{t \in \Omega}\left[s^{T}(t) R(t) s(t)\right]^{\frac{1}{2}} .
$$

\footnotetext{
${ }^{1}$ For the sake of brevity, we denote by $\mathcal{L}_{p}$ the set $\mathcal{L}_{p}([0,+\infty))$.
} 
When the weighting matrix $R(\cdot)$ is time-invariant and equal to the identity matrix $I$, we will use the simplified notation $\|s(\cdot)\|_{p}$.

\section{PROBLEM STATEMENT AND PRELIMINARY RESULTS}

In general, $w(\cdot) \in \mathcal{L}_{p}$ does not guarantee that $y(\cdot) \in \mathcal{L}_{p} ;$ therefore it makes sense to give the definition of IO $\mathcal{L}_{p^{-}}$ stability. Roughly speaking (the precise definition is more involved, the interested reader is referred to [11], Ch. 5, or to [1]), system (1) is said to be $\mathcal{L}_{p}$-stable, if $w(\cdot) \in \mathcal{L}_{p}$ implies $y(\cdot) \in \mathcal{L}_{p}$. The most popular cases are the ones with $p=2$ and $p=\infty$.

The concept of $\mathcal{L}_{p}$-stability is generally referred to an infinite interval of time. In this paper we are interested to study the input-output behavior of the system over a finite time interval.

Let us consider a linear time-varying (LTV) system in the form

$$
\Gamma:\left\{\begin{array}{l}
\dot{x}(t)=A(t) x(t)+G(t) w(t), \quad x\left(t_{0}\right)=0 \\
y(t)=C(t) x(t)
\end{array}\right.
$$

where $A(\cdot): \Omega \mapsto \mathbb{R}^{n \times n}, G(\cdot): \Omega \mapsto \mathbb{R}^{n \times r}$, and $C(\cdot): \Omega \mapsto \mathbb{R}^{m \times n}$, are continuous matrix-valued functions; $\Gamma$ can be viewed as a linear operator mapping input signals $(w(\cdot)$ 's) into output signals $(y(\cdot)$ 's).

In the following we will denote by $\Phi(t, \tau)$ the state transition matrix of system (1).

\section{A. Definition of IO-FTS}

In this section we introduce the concept of IO-FTS for the class of systems in the form (1). To this end, let us consider the following definition.

Definition 1 (IO-FTS of LTV systems): Given positive scalar $T$, a class of input signals $\mathcal{W}$ defined over $\Omega=\left[t_{0}, t_{0}+T\right]$, a continuous and positive definite matrix-valued function $Q(\cdot)$ defined in $\Omega$, system (1) is said to be IO-FTS with respect to $(\mathcal{W}, Q(\cdot), \Omega)$ if

$$
w(\cdot) \in \mathcal{W} \Rightarrow y^{T}(t) Q(t) y(t)<1, \quad t \in \Omega .
$$

In this paper we consider the class of norm bounded square integrable signals over $\Omega$, defined as follows

$$
\mathcal{W}_{2}(\Omega, R(\cdot)):=\left\{w(\cdot) \in \mathcal{L}_{2}(\Omega):\|w\|_{2, R} \leq 1\right\}
$$

where $R(\cdot)$ denotes a continuous positive definite matrixvalued function.

In the rest of the paper we will drop the dependency of $\mathcal{W}_{2}$ on $\Omega$ and $R(\cdot)$ in order to simplify the notation.

In Section III two necessary and sufficient conditions for IO-FTS for the class of $\mathcal{W}_{2}$ input signals are provided.

\section{B. Reachability Gramian}

We now introduce some preliminary results concerning the reachability Gramian of LTV systems, which are then exploited in this paper to prove the main result. More details can be found in [7].

Definition 2 (Reachability Gramian): The reachability Gramian of system (1) is defined as

$$
W_{r}\left(t, t_{0}\right) \triangleq \int_{t_{0}}^{t} \Phi(t, \tau) G(\tau) G^{T}(\tau) \Phi^{T}(t, \tau) d \tau .
$$

Note that $W_{r}\left(t, t_{0}\right)$ is symmetric and positive semidefinite for all $t \geq t_{0}$.

Lemma 1 ([7]): Given system (1), $W_{r}\left(t, t_{0}\right)$ is the unique solution of the matrix differential equation

$$
\dot{W}_{r}\left(t, t_{0}\right)=A(t) W_{r}\left(t, t_{0}\right)+W_{r}\left(t, t_{0}\right) A^{T}(t)+G(t) G^{T}(t),
$$

$W_{r}\left(t_{0}, t_{0}\right)=0$

\section{Preliminary Results}

In this section we state an equivalent definition of IOFTS that can be easily derived when the LTV system (1) is regarded as a linear operator that maps signals from the space $\mathcal{L}_{2}(\Omega)$ to the space $\mathcal{L}_{\infty}(\Omega)$, i.e.:

$$
\Gamma: w(\cdot) \in \mathcal{L}_{2}(\Omega) \mapsto y(\cdot) \in \mathcal{L}_{\infty}(\Omega) .
$$

Furthermore, if we equip the $\mathcal{L}_{2}(\Omega)$ and $\mathcal{L}_{\infty}(\Omega)$ spaces with the weighted norms $\|\cdot\|_{2, R}$ and $\|\cdot\|_{\infty, Q}$, respectively, the induced norm of the linear operator (3) is given by

$$
\|\Gamma\|=\sup _{\|w(\cdot)\|_{2, R}=1}\left[\|y(\cdot)\|_{\infty, Q}\right],
$$

that is the norm of $\Gamma$ is computed considering the input signals in $\mathcal{W}_{2}$. Hence, to require system (1) being IO-FTS wrt $\left(\mathcal{W}_{2}, Q(\cdot), \Omega\right)$ is equivalent to require that $\|\Gamma\|<1$; the following theorem holds.

Theorem 1: Given a time interval $\Omega$, the class of input signals $\mathcal{W}_{2}$, and a continuous positive definite matrixvalued function $Q(\cdot)$, system (1) is IO-FTS with respect to $\left(\mathcal{W}_{2}, Q(\cdot), \Omega\right)$ if and only if $\|\Gamma\|<1$.

Given the linear operator (3), its dual operator is

$$
\bar{\Gamma}: z(\cdot) \in \mathcal{L}_{1}(\Omega) \mapsto v(\cdot) \in \mathcal{L}_{2}(\Omega),
$$

corresponding to the dual system [7, pg. 236]

$$
\bar{\Gamma}:\left\{\begin{array}{l}
\dot{\tilde{x}}(t)=-A^{T}(t) \tilde{x}(t)-C^{T}(t) z(t) \\
v(t)=G^{T}(t) \tilde{x}(t)
\end{array} .\right.
$$

According to duality, the norm of $\bar{\Gamma}$ is defined as

$$
\|\bar{\Gamma}\|=\sup _{\|z(\cdot)\|_{1, Q}=1}\left[\|v(\cdot)\|_{2, R}\right] ;
$$

moreover, by definition of dual operator ([14]) we have that, given $z(\cdot) \in \mathcal{L}_{1}(\Omega)$ and $w(\cdot) \in \mathcal{L}_{2}(\Omega)$,

$$
\langle z, \Gamma w\rangle=\langle\bar{\Gamma} z, w\rangle
$$


where, given two signals $u(\cdot)$ and $v(\cdot)$, we have

$$
\langle u, v\rangle=\int_{\Omega} u^{T}(t) v(t) d t .
$$

Furthermore it holds that (see [14, p. 195]):

$$
\|\Gamma\|=\|\bar{\Gamma}\| \text {. }
$$

The next theorem is a generalization of a result given in [13] to the case of LTV systems, and it allows us to compute the norm of $\Gamma$ as a function of the spectral radius of the reachability Gramian defined in Section II-B.

Theorem 2: Given the LTV system (1), the norm of the corresponding linear operator (3) is given by

$$
\|\Gamma\|=\operatorname{ess} \sup _{t \in \Omega} \lambda_{\max }^{\frac{1}{2}}\left(Q^{\frac{1}{2}}(t) C(t) W\left(t, t_{0}\right) C^{T}(t) Q^{\frac{1}{2}}(t)\right),
$$

for all $t \in \Omega$, where $\lambda_{\max }(\cdot)$ denotes the maximum eigenvalue, and $W\left(t, t_{0}\right)$ is the positive semidefinite matrixvalued solution of

$$
\begin{aligned}
\dot{W}\left(t, t_{0}\right) & =A(t) W\left(t, t_{0}\right)+W\left(t, t_{0}\right) A^{T}(t) \\
& +G(t) R(t)^{-1} G^{T}(t) \\
W\left(t_{0}, t_{0}\right) & =0
\end{aligned}
$$

Proof. For the sake of simplicity, we consider the weighting matrices equal to the identity, hence

$$
R(t)=I \text { and } Q(t)=I, \forall t \in \Omega .
$$

Note that, given this assumption, the solution of (8) is given by the reachability gramian $W_{r}\left(t, t_{0}\right)$; we will discuss how to take into account the weighting matrices at the end of the proof.

First note that, in view of (6), proving (7) is equivalent to show that

$$
\|\bar{\Gamma}\|=\operatorname{ess} \sup _{t \in \Omega} \lambda_{\max }^{\frac{1}{2}}\left(C(t) W_{r}\left(t, t_{0}\right) C^{T}(t)\right),
$$

where $\bar{\Gamma}$ is the dual operator of $\Gamma$. Taking into account the definition of $\bar{\Gamma}$, let us denote by

$$
\bar{H}(t, \tau)=G^{T}(t) \Phi^{T}(\tau, t) C^{T}(\tau) \delta_{-1}(\tau-t)
$$

the impulsive response of the dual system (4), where $\delta_{-1}(t)$ is the Heaviside step function. Letting

$$
\Upsilon(t)=\int_{\Omega} \bar{H}^{T}(t, \tau) \bar{H}(\tau, t) d t,
$$

if $v(\cdot)$ is the output of system (4), we have

$$
\begin{aligned}
& \|v(\cdot)\|_{2}=\left\|\int_{\Omega} \bar{H}(\cdot, \tau) z(\tau) d \tau\right\|_{2} \\
& \leq \int_{\Omega}\|\bar{H}(\cdot, \tau) z(\tau)\|_{2} d \tau \\
& =\int_{\Omega}\left(z^{T}(\tau) \Upsilon(\tau) z(\tau)\right)^{\frac{1}{2}} d \tau \text { by (9) } \\
& \leq \int_{\Omega}\left|\Upsilon(\tau)^{\frac{1}{2}}\right| \cdot|z(\tau)| d \tau \\
& =\int_{\Omega} \lambda_{\max }^{\frac{1}{2}}(\Upsilon(\tau)) \cdot|z(\tau)| d \tau \\
& \leq \operatorname{ess} \sup _{t \in \Omega} \lambda_{\max }^{\frac{1}{2}}(\Upsilon(t)) \cdot \int_{\Omega}|z(\tau)| d \tau \\
& =\operatorname{ess}_{\sup _{t \in \Omega}} \lambda_{\max }^{\frac{1}{2}}(\Upsilon(t)) \cdot\|z(\cdot)\|_{1},
\end{aligned}
$$

thus

$$
\|\bar{\Gamma}\| \leq \operatorname{ess} \sup _{t \in \Omega} \lambda_{\max }^{\frac{1}{2}}(\Upsilon(t)) .
$$

From Definition 2 the matrix-valued function $\Upsilon(t)$ is equal to

$$
\Upsilon(t)=C(t) W_{r}\left(t, t_{0}\right) C^{T}(t) ;
$$

hence (10) reads

$$
\|\bar{\Gamma}\| \leq \operatorname{ess} \sup _{t \in \Omega} \lambda_{\max }^{\frac{1}{2}}\left(C(t) W_{r}\left(t, t_{0}\right) C^{T}(t)\right) .
$$

The last part of the proof is devoted to show that (11) is actually an equality. In order to do that, let

$$
\text { ess } \sup _{t \in \Omega} \lambda_{\max }^{\frac{1}{2}}[\Upsilon(t)]=\gamma,
$$

therefore (11) reads

$$
\|\bar{\Gamma}\| \leq \gamma .
$$

In the following we shall build a sequence of inputs to system (4) with unit norm in $\mathcal{L}_{1}(\Omega)$, such that the sequence of the norms of the corresponding output signals converges to $\gamma$.

To this end consider a subset $\Omega^{\prime} \subset \Omega$, such that, for all $t \in \Omega^{\prime}$,

$$
\lambda_{\max }^{\frac{1}{2}}\left(C(t) W_{r}\left(t, t_{0}\right) C^{T}(t)\right) \geq \gamma-\varepsilon,
$$

with $\varepsilon>0$. Now let $\sigma \in \Omega^{\prime}$ and consider the sequence of inputs

$$
z_{\varepsilon, \alpha}(t)=h(\sigma) u_{\alpha}(t)
$$

where $h(\sigma)$ is the unit eigenvector corresponding to the maximum eigenvalue of $C(\sigma) W_{r}\left(\sigma, t_{0}\right) C^{T}(\sigma)$, and $u_{\alpha}$ is a sequence of positive scalar functions with unit norm in $\mathcal{L}_{1}(\Omega)$, which approach the Dirac delta function applied in $\sigma$ as $\alpha \mapsto 0$. Let

$$
v_{\varepsilon, \alpha}(t)=\bar{\Gamma} z_{\varepsilon, \alpha}(t)=\int_{\Omega} \bar{H}(t, \tau) z_{\varepsilon, \alpha}(\tau) d \tau,
$$

then it is

$$
\lim _{\alpha \rightarrow 0}\left\|v_{\varepsilon, \alpha}(\cdot)\right\|_{2}^{2}=h^{T}(\sigma) C(\sigma) W_{r}\left(\sigma, t_{0}\right) C^{T}(\sigma) h(\sigma),
$$

and therefore we can conclude that

$$
\lim _{\alpha \mapsto 0}\left\|v_{\varepsilon, \alpha}(\cdot)\right\|_{2}=\lambda_{\max }^{\frac{1}{2}}\left(C(\sigma) W_{r}\left(\sigma, t_{0}\right) C^{T}(\sigma)\right) \geq \gamma-\varepsilon .
$$

Hence, given $\eta>0$, it is possible to choose a sufficiently small $\alpha$ such that

$$
\left\|v_{\varepsilon, \alpha}(\cdot)\right\|_{2} \geq \gamma-\varepsilon-\eta .
$$

Taking into account (12), that the scalars $\varepsilon$ and $\eta$ can be chosen arbitrarily small, and that the set of the signals $z_{\varepsilon, \alpha}$ is a subset of the set of the unit norm signals in $\mathcal{L}_{1}(\Omega)$, we can conclude that

$$
\begin{aligned}
\gamma \geq\|\bar{\Gamma}\| & =\sup _{\|z(\cdot)\|_{1}=1}\|v(\cdot)\|_{2} \\
& \geq \sup _{z_{\varepsilon, \alpha}(\cdot)}\left\|v_{\varepsilon, \alpha}(\cdot)\right\|_{2}=\gamma
\end{aligned}
$$

From the last chain of inequality the proof follows. 
Eventually, note that when the weighting matrices are taken into account the proof still holds by modifying the model matrices as follows

$$
\widetilde{G}(t)=G(t) R(t)^{-\frac{1}{2}}, \quad \widetilde{C}(t)=Q^{\frac{1}{2}}(t) C(t),
$$

and replacing $W_{r}\left(t, t_{0}\right)$ by $W\left(t, t_{0}\right)$.

Remark 1: It is worth to notice that, since all the system matrices in (1) and the weighting matrices $R(\cdot)$ and $Q(\cdot)$ are assumed to be continuous, in the closed time interval $\Omega$ the condition (7) is equivalent to

$$
\|\Gamma\|=\max _{t \in \Omega} \lambda_{\max }^{\frac{1}{2}}\left(Q^{\frac{1}{2}}(t) C(t) W\left(t, t_{0}\right) C^{T}(t) Q^{\frac{1}{2}}(t)\right) .
$$

We conclude the section with the following technical lemma, which will be useful to prove the main result of the paper in Section III.

Lemma 2: Given $\epsilon>0$, the solution of the matrix differential equation

$$
\begin{aligned}
\dot{W}_{\epsilon}\left(t, t_{0}\right) & =A(t) W_{\epsilon}\left(t, t_{0}\right)+W_{\epsilon}\left(t, t_{0}\right) A^{T}(t) \\
& +G(t) R(t)^{-1} G^{T}(t)+\epsilon I, \\
W_{\epsilon}\left(t_{0}, t_{0}\right) & =\epsilon I
\end{aligned}
$$

is the positive definite matrix

$$
\begin{aligned}
W_{\epsilon}\left(t, t_{0}\right)=W\left(t, t_{0}\right)+ & \epsilon \Phi\left(t, t_{0}\right) \Phi^{T}\left(t, t_{0}\right) \\
& +\epsilon \int_{t_{0}}^{t} \Phi(t, \tau) \Phi^{T}(t, \tau) d \tau,
\end{aligned}
$$

where $W(\cdot, \cdot)$ is the solution of equations (8).

Proof: The proof follows from direct substitution of $W_{\epsilon}(\cdot, \cdot)$ in (13), and by the fact that the matrix $\Phi\left(t, t_{0}\right) \Phi^{T}\left(t, t_{0}\right)$ is positive definite.

\section{MAIN RESULT}

The main result of this section is the following theorem that states two necessary and sufficient conditions for the IO-FTS of system (1).

Theorem 3: Given system (1), the class of inputs $\mathcal{W}_{2}$, a continuous positive definite matrix-valued function $Q(\cdot)$, and the time interval $\Omega$, the following statements are equivalent:

i) System (1) is IO-FTS with respect to $\left(\mathcal{W}_{2}, Q(\cdot), \Omega\right)$.

ii) The inequality

$$
\lambda_{\max }\left(Q^{\frac{1}{2}}(t) C(t) W\left(t, t_{0}\right) C^{T}(t) Q^{\frac{1}{2}}(t)\right)<1
$$

holds for all $t \in \Omega$, where $W(\cdot, \cdot)$ is the positive semidefinite solution of the Differential Lyapunov Equality (DLE) (8).

iii) The coupled DLMI/LMI

$$
\begin{array}{cc}
\left(\begin{array}{cc}
\dot{P}(t)+A^{T}(t) P(t)+P(t) A(t) & P(t) G(t) \\
G^{T}(t) P(t) & -R(t)
\end{array}\right)<0 \\
P(t)>C^{T}(t) Q(t) C(t),
\end{array}
$$

admits a positive definite solution $P(\cdot)$ over $\Omega$.
Proof. We will prove the equivalence of the three statements by showing that i) $\Rightarrow$ ii), ii) $\Rightarrow$ iii), and iii) $\Rightarrow$ i).

ii) $\Rightarrow$ ii)]. The proof readily follows from Theorems 1 and 2, and from Remark 1.

[ii) $\Rightarrow$ iii)]. Given $\epsilon>0$, consider the DLE (13), whose solution $W_{\epsilon}(\cdot, \cdot)$, given by (14), is positive definite and satisfies the DLMI

$$
\begin{aligned}
-\dot{W}_{\epsilon}\left(t, t_{0}\right)+A(t) W_{\epsilon}( & \left.t, t_{0}\right)+W_{\epsilon}\left(t, t_{0}\right) A^{T}(t) \\
& +G(t) R(t)^{-1} G^{T}(t)<0 .
\end{aligned}
$$

Now letting

$$
W_{\epsilon}\left(t, t_{0}\right)=P^{-1}(t),
$$

it follows that $\dot{W}_{\epsilon}\left(t, t_{0}\right)=-P^{-1}(t) \dot{P}(t) P^{-1}(t)$, and inequality (17) reads

$$
\begin{array}{r}
P^{-1}(t) \dot{P}(t) P^{-1}(t)+A(t) P^{-1}(t)+P^{-1}(t) A^{T}(t) \\
+G(t) R^{-1}(t) G^{T}(t)<0,
\end{array}
$$

for all $t \in \Omega$. By pre- and post-multiply (18) by $P(t)$ we obtain

$$
\begin{aligned}
\dot{P}(t)+P(t) A(t) & +A^{T}(t) P(t) \\
& +P(t) G(t) R^{-1}(t) G^{T}(t) P(t)<0,
\end{aligned}
$$

and (16a) readily follows by applying Schur complements.

In order to prove (16b), first note that $W_{\epsilon}(\cdot, \cdot) \stackrel{\epsilon \mapsto 0}{\longrightarrow} W(\cdot, \cdot)$, hence, by continuity arguments, there exists a sufficiently small $\epsilon$ such that

$$
\lambda_{\max }\left(Q^{\frac{1}{2}}(t) C(t) W_{\epsilon}\left(t, t_{0}\right) C^{T}(t) Q^{\frac{1}{2}}(t)\right)<1 .
$$

Furthermore, condition (20) is equivalent to

$$
I-Q^{\frac{1}{2}}(t) C(t) P^{-1}(t) C^{T}(t) Q^{\frac{1}{2}}(t)>0,
$$

that, by applying Schur complements, reads

$$
\left(\begin{array}{cc}
I & Q^{\frac{1}{2}}(t) C(t) \\
C^{T}(t) Q^{\frac{1}{2}}(t) & P(t)
\end{array}\right)>0 .
$$

From [2, Lemma 5.3] inequality (22) is equivalent to

$$
\left(\begin{array}{cc}
P(t) & C^{T}(t) Q^{\frac{1}{2}}(t) \\
Q^{\frac{1}{2}}(t) C(t) & I
\end{array}\right)>0,
$$

which yields (16b) by applying again Schur complements.

$[$ iii) $\Rightarrow$ i)] . In [3] it has been proven that conditions (16) imply IO-FTS when the class of $\mathcal{W}_{2}$ signals is considered.

As a conclusion of this section, we want to remark that from Theorem 3 it readily follows that the conditions given in [3, Th. 3] are also necessary. 
TABLE I

MAXimum VALUes of $Q$ SATISFYing THEOREM 3 FOR THE LTV System (23).

\begin{tabular}{|c|c|c|c|}
\hline IO-FTS condition & Sample Time $\left(T_{s}\right)$ & Estimate of $Q_{\max }$ & Computation time [s] \\
\hline \hline \multirow{3}{*}{ DLMI (16) } & 0.05 & 0.2 & 2.5 \\
\cline { 2 - 4 } & 0.025 & 0.25 & 12.7 \\
\cline { 2 - 4 } & 0.0125 & 0.29 & 257 \\
\cline { 2 - 4 } & 0.00833 & 0.3 & 1259 \\
\hline \hline Solution of (8) and inequality (15) & 0.003 & 0.345 & 6 \\
\hline
\end{tabular}

\section{NUMERICAL EXAMPLE}

This section shows the effectiveness of the proposed approach to check the IO-FTS of LTV systems such as (1), when the input signal belong to class $\mathcal{W}_{2}$.

Let us consider the system

$$
\begin{aligned}
A(t)=\left(\begin{array}{cc}
0.5+t & 0.1 \\
0.4 & -0.3+t
\end{array}\right), G & =\left(\begin{array}{l}
1 \\
1
\end{array}\right), \\
C & =\left(\begin{array}{ll}
1 & 1
\end{array}\right),
\end{aligned}
$$

together with the following IO-FTS parameters:

$$
R=1, \Omega=[0,0.5] \text {. }
$$

The conditions stated in Theorem 3 are, in principle, necessary and sufficient. However, due to the time-varying nature of the involved matrices, the numerical implementation of such conditions introduces some conservativeness.

In order to compare each other, from the computational point of view, the conditions stated in Theorem 3, the output weighting matrix is left as a free parameter. More precisely, we introduce the parameter $Q_{\max }$, defined as the maximum value of the matrix $Q$ such that system (23) is IO-FTS, and use the conditions stated in Theorem 3 to obtain an estimate of $Q_{\max }$.

To recast the DLMI condition (16) in terms of LMIs, the matrix-valued functions $P(\cdot)$ has been assumed piecewise linear. In particular, the time interval $\Omega$ has been divided in $n=T / T_{s}$ subintervals, and the time derivatives of $P(t)$ have been considered constant in each subinterval. It is straightforward to recognize that such a piecewise linear function can approximate at will a given continuous matrix function, provided that $T_{s}$ is sufficiently small.

Given a piecewise linear function $P(\cdot)$, the feasibility problem (16) has been solved by exploiting standard optimization tools such as the Matlab LMI Toolbox ${ }^{\circledR}$ [9].

Since the equivalence between IO-FTS and condition (16) holds when $T_{s} \mapsto 0$, the maximum value of $Q$ satisfying condition (16), namely $Q_{\max }$, has been evaluated for different values of $T_{s}$. The obtained estimates of $Q_{\max }$, the corresponding values of $T_{s}$ and of the computation time are shown in Table I. These results have been obtained by using a PC equipped with an Intel ${ }^{\circledR}$ i7-720QM processor and 4 GB of RAM.

We have then considered the problem of finding the maximum value of $Q$ satisfying condition (15), where $W(\cdot, \cdot)$ is the positive semidefinite solution of (8). In particular, equation (8) has been firstly integrated, with a sample time $T_{s}=0.003 \mathrm{~s}$, by using the Euler forward method, and then the maximum value of $Q$ satisfying condition (15) has been evaluated by means of a linear search. As a result, it has been found the estimate $Q_{\max }=0.345$, with a computation time of about $6 \mathrm{~s}$, as it is shown in the last row of Table I.

We can conclude that the condition based on the reachability Gramian is much more efficient with respect to the solution of the DLMI when considering the IO-FTS analysis problem.

\section{CONCLUSIONS}

Necessary and sufficient conditions for IO-FTS are presented in this paper for the class of $\mathcal{W}_{2}$ input signals. The proof of the main result is based on Reachability Gramian theory, and the necessary and sufficient condition is provided in terms of DLMI feasibility problem. The effectiveness of the proposed result has been shown by means of a numerical example.

\section{REFERENCES}

[1] C. T. Abdallah, F. Amato, and M. Ariola. Input-output stability. In Encyclopedia of Electrical and Electronics Engineering. Wiley, 2001.

[2] F. Amato. Robust Control of Linear Systems Subject to Uncertain Time-Varying Parameters. Springer Verlag, 2006.

[3] F. Amato, R. Ambrosino, C. Cosentino, and G. De Tommasi. Input-output finite-time stabilization of linear systems. Automatica, 46(9):1558-1562, Sep. 2010.

[4] F. Amato, M. Ariola, and C. Cosentino. Finite-time stabilization via dynamic output feedback. Automatica, 42:337-342, 2006.

[5] F. Amato, M. Ariola, and P. Dorato. Finite-time control of linear systems subject to parametric uncertanties and disturbances. Automatica, 37:1459-1463, 2001.

[6] R. Ambrosino, F. Calabrese, C. Cosentino, and G. De Tommasi. Sufficient conditions for finite-time stability of impulsive dynamical systems. IEEE Trans. on Auto. Contr., 54(4):364-369, Apr. 2009.

[7] F. Callier and C. A. Desoer. Linear System Theory. Springer Verlag, 1991.

[8] P. Dorato. Short time stability in linear time-varying systems. In Proc. IRE International Convention Record Part 4, pages 83-87, 1961.

[9] P. Gahinet, A. Nemirovski, A. J. Laub, and M. Chilali. LMI Control Toolbox. The Mathworks Inc, 1995.

[10] G. Garcia, S. Tarbouriech, and J. Bernussou. Finite-time stabilization of linear time-varying continuous systems. IEEE Trans. on Auto. Contr., 54(4):861-865, Apr. 2009.

[11] H. K. Khalil. Nonlinear Systems. Prentice Hall, $3^{\text {rd }}$ edition, 2001.

[12] Y. Shen. Finite-time control of linear parameter-varying systems with norm-bounded exogenous disturbance. J. Contr. Theory Appl., 6(2):184-188, 2008.

[13] D. A. Wilson. Convolution and hankel operator norms for linear systems. IEEE Trans. on Auto. Contr., 34(1):94-97, 1989.

[14] K. Yosida. Functional Analysis. Springer Verlag, 1980.

[15] S. Zhao, J. Sun, and L. Liu. Finite-time stability of linear time-varying singular systems with impulsive effects. Int. J. Control, 81(11):18241829,2008 